\title{
BMJ Open Heavier smoking may lead to a relative increase in waist circumference: evidence for a causal relationship from a Mendelian randomisation meta-analysis. The CARTA consortium
}

Richard W Morris, ${ }^{1,2}$ Amy E Taylor, ${ }^{3,4}$ Meg E Fluharty, ${ }^{3,4}$ Johan H Bjørngaard, Bjørn Olav Åsvold, ${ }^{5,7}$ Maiken Elvestad Gabrielsen, ${ }^{8}$ Archie Campbell, ${ }^{9}$ Riccardo Marioni, ${ }^{9,10,11}$ Meena Kumari, ${ }^{12}$ Tellervo Korhonen, ${ }^{13,14,15}$ Satu Männistö, ${ }^{13}$ Pedro Marques-Vidal, ${ }^{16}$ Marika Kaakinen, ${ }^{17,18}$ Alana Cavadino, ${ }^{19,20}$ Iris Postmus, ${ }^{21,22}$ Lise Lotte N Husemoen, ${ }^{23}$ Tea Skaaby, ${ }^{23}$ Tarun Veer Singh Ahluwalia, 24,25,26 Jorien L Treur, ${ }^{27}$ Gonneke Willemsen, ${ }^{27}$ Caroline Dale, ${ }^{28} \mathrm{~S}$ Goya Wannamethee, ${ }^{2}$ Jari Lahti, ${ }^{29,30}$ Aarno Palotie, ${ }^{31,32,33}$ Katri Räikkönen, ${ }^{29}$ Alex McConnachie, ${ }^{34}$ Sandosh Padmanabhan, ${ }^{35}$ Andrew Wong ${ }^{36}$ Christine Dalgård, ${ }^{37}$ Lavinia Paternoster, ${ }^{1,3}$ Yoav Ben-Shlomo, ${ }^{1}$ Jessica Tyrrell, ${ }^{38,39}$ John Horwood, ${ }^{40}$ David M Fergusson, ${ }^{40}$ Martin A Kennedy, ${ }^{41}$ Ellen A Nohr, ${ }^{42}$ Lene Christiansen, ${ }^{43}$ Kirsten Ohm Kyvik, ${ }^{37}$ Diana Kuh, ${ }^{36}$ Graham Watt, ${ }^{44}$ Johan G Eriksson, ${ }^{15,30,45,46,47}$ Peter H Whincup, ${ }^{48}$ Jacqueline M Vink, ${ }^{27}$ Dorret I Boomsma, ${ }^{27}$ George Davey Smith, ${ }^{1,3}$ Debbie Lawlor, ${ }^{1,3}$ Allan Linneberg, ${ }^{23,49,50}$ Ian Ford, ${ }^{34} \mathrm{~J}$ Wouter Jukema, ${ }^{51,52,53}$ Chris Power, ${ }^{20}$ Elina Hyppönen, ${ }^{20,54,55}$ Marjo-Riitta Jarvelin, ${ }^{17,18,56,57,58}$ Martin Preisig, ${ }^{59}{ }^{\prime}$ Katja Borodulin, ${ }^{15}$ Jaakko Kaprio, ${ }^{13,15,60}$ Mika Kivimaki, ${ }^{61}$ Blair $\mathrm{H}$ Smith, ${ }^{62}$ Caroline Hayward, ${ }^{63}$ Pål R Romundstad, ${ }^{5}$ Thorkild I A Sørensen, ${ }^{3,24,64}$ Marcus R Munafò,, ${ }^{3,4}$ Naveed Sattar ${ }^{65}$

To cite: Morris RW,

Taylor AE, Fluharty ME, et al. Heavier smoking may lead to a relative increase in waist circumference: evidence for a causal relationship from a Mendelian randomisation meta-analysis. The CARTA consortium. BMJ Open 2015;5:e008808.

doi:10.1136/bmjopen-2015008808

\section{- Prepublication history and} additional material is available. To view please visit the journal (http://dx.doi.org/ 10.1136/bmjopen-2015008808).

Received 18 May 2015 Revised 14 July 2015 Accepted 21 July 2015

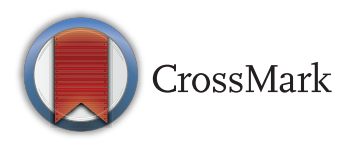

For numbered affiliations see end of article.

Correspondence to

Professor Richard W Morris; richard.morris@bristol.ac.uk

\section{ABSTRACT}

Objectives: To investigate, using a Mendelian randomisation approach, whether heavier smoking is associated with a range of regional adiposity phenotypes, in particular those related to abdominal adiposity.

Design: Mendelian randomisation meta-analyses using a genetic variant (rs16969968/rs1051730 in the CHRNA5-CHRNA3-CHRNB4 gene region) as a proxy for smoking heaviness, of the associations of smoking heaviness with a range of adiposity phenotypes.

Participants: 148731 current, former and neversmokers of European ancestry aged $\geq 16$ years from 29 studies in the consortium for Causal Analysis Research in Tobacco and Alcohol (CARTA).

Primary outcome measures: Waist and hip circumferences, and waist-hip ratio.

Results: The data included up to 66809 never-smokers, 43009 former smokers and 38913 current daily cigarette smokers. Among current smokers, for each extra minor allele, the geometric mean was lower for waist circumference by $-0.40 \%(95 \% \mathrm{Cl}-0.57 \%$ to $-0.22 \%$ ), with effects on hip circumference, waist-hip ratio and body mass index (BMI) being $-0.31 \%(95 \% \mathrm{Cl}$ $-0.42 \%$ to -0.19$),-0.08 \%(-0.19 \%$ to $0.03 \%)$ and $-0.74 \%(-0.96 \%$ to $-0.51 \%)$, respectively. In contrast, among never-smokers, these effects were higher by $0.23 \%(0.09 \%$ to $0.36 \%), 0.17 \%$ ( $0.08 \%$ to $0.26 \%)$, $0.07 \%(-0.01 \%$ to $0.15 \%)$ and $0.35 \%(0.18 \%$ to $0.52 \%$ ), respectively. When adjusting the three central adiposity measures for BMI, the effects among current smokers changed direction and were higher by $0.14 \%$

\section{Strengths and limitations of this study}

- This is a very large Mendelian randomisation study of the relationship between smoking and several anthropometric phenotypes relating to regional adiposity.

- Data included never, former and current smokers from a very wide spectrum of ages among 29 studies.

- By using a genetic variant associated with smoking heaviness as a proxy for smoking heaviness, bias from confounding is minimised and findings are not affected by reverse causality.

- Data for direct measures of fat, such as fat mass, and the biomarker leptin were available for only about one fifth of the participants whose weight, height, waist and hip were measured.

- Participants were exclusively of self-reported European ancestry, and were mostly recruited in European countries.

( $0.05 \%$ to $0.22 \%$ ) for waist circumference, $0.02 \%$ ( $-0.05 \%$ to $0.08 \%)$ for hip circumference and $0.10 \%$ $(0.02 \%$ to $0.19 \%)$ for waist-hip ratio, for each extra minor allele.

Conclusions: For a given BMI, a gene variant associated with increased cigarette consumption was associated with increased waist circumference. Smoking in an effort to control weight may lead to accumulation of central adiposity. 


\section{INTRODUCTION}

Tobacco is the single most important cause of preventable death globally: one in two young people taking up lifelong cigarette smoking will die of causes related to it. ${ }^{1}$ Enormous efforts have gone into developing interventions for smoking cessation. Spontaneous cessation rates are low due to the high proportion of smokers who are dependent on nicotine, and effective treatments are still not widely available. One barrier to smoking cessation is the fear of weight gain. In a study of almost 2000 smokers in the USA, recruited into a trial of bupropion and/or nicotine inhalers to promote cessation, $50 \%$ of female and $26 \%$ of male smokers reported that gaining weight discouraged them from trying to quit, ${ }^{2}$ while among adults in Finland, daily smokers were found to report more weight concerns than former smokers or occasional smokers. ${ }^{3}$

A genetic variant in the chromosome 15 CHRNA5CHRNA3-CHRNB4 gene region (rs16969968) codes for a functional amino acid change D398N in the nicotinic receptor $\alpha 5$ subunit. The SNP rs16969968, which is in perfect linkage disequilibrium with SNP rs1051730 in European populations, is associated with smoking quantity among smokers. ${ }^{4}$ The minor allele of this variant is associated with an average increase in smoking amount of one cigarette per day in smokers and increases in cotinine (a metabolite of nicotine) levels. ${ }^{5}{ }^{6}$ It has also been found that the variant was associated with a lower mean body mass index (BMI) ${ }^{7-9}$ thus adding evidence that heavier smoking leads to lower BMI. The latter study also noted lower waist and hip circumferences among smokers with the variant. ${ }^{8}$ However, prior observational evidence suggests that waist circumference and waist-hip ratio may be higher in smokers than in non-smokers after adjusting for BMI. ${ }^{10}$ It has also been observed that smoking in adolescence predicts abdominal obesity in adulthood. ${ }^{11}$ Moreover, heavy smokers exhibit greater central adiposity than light smokers, based on an analysis of middle-aged smokers of European ancestry. ${ }^{12}$ These studies suggest that smoking leads to a central fat accumulation at the expense of peripheral fat loss, particularly in women. ${ }^{13}$ In addition, there are also suggestions that smoking may lead to loss of muscle mass as indicated by lower hip circumferences in smokers. This is of high public health relevance in view of the reportedly greater impact of increased central adiposity both on mortality ${ }^{14} 15$ and on the development of diabetes, especially among women, ${ }^{16}{ }^{17}$ and since smoking is associated with an increased risk of type 2 diabetes. ${ }^{18}$

We previously used Mendelian randomisation methods to investigate the effect of smoking quantity on BMI. ${ }^{79}$ This method exploits Mendel's laws concerning the random assortment of alleles at the time of gamete formation so that individuals are allocated at random to having 0,1 or 2 alleles in the rs1051730/rs16969968 genotype. The effect of this genotype on smoking quantity among smokers has been demonstrated, ${ }^{6}$ and thus the inverse relationship between allele count and BMI is not subject to effects of confounding and reverse causality. Using a substantial pool of studies in the consortium for Causal Analysis Research in Tobacco and Alcohol (CARTA), we have extended our use of Mendelian randomisation methods to examine the effect of smoking quantity on a range of adiposity phenotypes. We test the hypotheses that (1) phenotypes representing central adiposity are affected by smoking quantity differentially from other phenotypes, and (2) these effects are more marked among women than among men.

\section{METHODS}

\section{Study populations}

We used data on individuals ( $\geq 16$ years) of self-reported European ancestry from 29 studies from the CARTA consortium (http://www.bris.ac.uk/expsych/research/ brain/targ/research/collaborations/carta/): the 1958 Birth Cohort (1958BC), the Avon Longitudinal Study of Parents and Children (ALSPAC, including both mothers and children), the British Regional Heart Study (BRHS), the British Women's Heart and Health Study (BWHHS), the Caerphilly Prospective Study (CaPS), the Christchurch Health and Development Study (CHDS), CoLaus, the Danish Monica study (Dan-MONICA), the Exeter Family Study of Child Health (EFSOCH), the English Longitudinal Study of Ageing (ELSA), the National FINRISK studies, GEMINAKAR, GS:SFHS (Generation Scotland: Scottish Family Health Study), the Genomics of Overweight Young Adults (GOYA) females, GOYA males, the Helsinki Birth Cohort Study (HBCS), Health2006, Health2008, the Nord-Trøndelag Health Study (HUNT), Inter99, MIDSPAN, the Northern Finland Birth Cohorts (NFBC 1966 and NFBC 1986), the National Health and Nutrition Examination Survey (NHANES), the MRC National Survey of Health \& Development (NSHD), the Netherlands Twin Register (NTR), the PROspective Study of Pravastatin in the Elderly at Risk (PROSPER) and Whitehall II. All studies received ethics approval from the local research ethics committees. Further details of these studies are provided in online supplementary material.

\section{Genotype}

Within each study, individuals were genotyped for one of two single nucleotide polymorphisms (SNPs) in the CHRNA5-A3-B4 nicotinic receptor subunit gene cluster, either rs 16969968 or rs1051730. These SNPs are in perfect linkage disequilibrium with each other in Europeans $\left(\mathrm{R}^{2}=1.00\right.$ in HapMap 3, http://hapmap.ncbi.nlm.nih.gov/ ) and therefore represent the same genetic signal. Where studies had data available for both SNPs, we used the SNP that was genotyped in the largest number of individuals. Details of genotyping methods within each study are provided in online supplementary material.

\section{Adiposity measures}

Direct physical measurements included weight, height, waist and hip circumferences, arm circumference, 
triceps skinfold and subscapular skinfold thickness. Fat mass and fat-free mass were available from bioimpedance measures, while leptin and adiponectin were the two biochemical markers related to fat mass.

BMI (weight/ $/$ height $^{2}$ ) and waist-hip ratio (waist/hip) were calculated.

Waist circumference and waist-hip ratio were taken as key measures of central adiposity, while BMI acted as a non-specific measure of adiposity for purposes of adjustment in regression analysis.

\section{Smoking status}

Smoking status was self-reported (either by questionnaire or interview) at the same time as regional adiposity measures for all studies, with the exception of 1958 BC (see online supplementary material). Individuals were classified as current, former, ever (ie, current and former combined) or never cigarette smokers. Where information on pipe and cigar smoking was available, individuals reporting being current or former smokers of pipes or cigars but not cigarettes were excluded from all analyses.

For studies with adolescent populations (ALSPAC children and NFBC 1986), analyses were restricted to current daily smokers who reported smoking at least one cigarette per day (current smokers) and individuals who had never tried smoking (never-smokers).

\section{Statistical analysis}

Analyses were conducted within each contributing study using Stata (Stata Corp, College Station, Texas, USA) and R (R Foundation for Statistical Computing, Vienna, Austria. http://www.R-project.org) software, following the same analysis plan. Analyses were restricted to individuals with full data on smoking status and rs1051730/ rs16969968 genotype, and having data on at least one of the regional adiposity phenotypes.

Within each study, genotype frequencies were tested for deviation from the Hardy Weinberg Equilibrium (HWE) using a $\chi^{2}$ test. Mendelian randomisation analyses of the association between rs1051730/rs16969968 and each regional adiposity phenotype were performed using linear regression, stratified by smoking status (never, former and current) and sex, and adjusted for age. Apart from height, natural logarithmic transforms were taken of every anthropometric phenotype. An additive genetic model was assumed on log values, so that each effect size could be exponentiated to represent the percentage increase per minor (risk) allele. These analyses were presented separately for each smoking status category. All phenotypic measures were further adjusted for $\log (\mathrm{BMI})$ (apart from weight, height and BMI itself), thus assessing the effect of the particular adiposity measure after adjusting for this global weight measure. $\log$ (weight) was adjusted for height instead of $\log (\mathrm{BMI})$. Since adjustment for ratio variables in anthropometric studies has been criticised, ${ }^{19}$ we further adjusted waist circumference for $\log$ (weight) and height.
Finally, we repeated analysis of waist circumference adjusted for BMI restricted to participants with BMI under $30 \mathrm{~kg} / \mathrm{m}^{2} ; 95 \%$ CIs have been quoted for all effect sizes.

Meta-analysis was also carried out of the relationship between reported daily cigarette consumption and rs1051730/rs16969968 genotype, among current smokers.

Although analyses were carried out separately for males and females, the estimates were combined where no evidence for separate sex effects was seen. For NHANES, which has a survey design, Taylor series linearisation was implemented to estimate variances. For studies including related family members, appropriate methods were used to adjust SEs: in GEMINAKAR, twin pair identity was included as a cluster variable in the model; in MIDSPAN, linear mixed effects regression models fitted using restricted maximum likelihood were used to account for related individuals, while in NTR, only unrelated individuals were included. ALSPAC mothers and children were analysed as separate samples; as there are related individuals across these samples, sensitivity analyses were performed excluding each of these studies in turn.

Results from individual studies were meta-analysed in Stata (V.13) using the 'metan' command from Stata. Where there was evidence of heterogeneity between studies $\left(\mathrm{I}^{2}>50 \%\right)$, it was planned that both fixed and random effects analyses would be performed: however, as this never occurred, results for fixed effects analysis only are shown. Meta-regression analysis, using the 'metareg' command from Stata, was used to examine whether SNP effects varied by smoking status or by sex, or by a smoking by sex combination.

\section{RESULTS}

\section{Descriptive statistics}

The maximum sample size available, with genotype recorded, was 148731 for weight, height and BMI over 29 studies. The data on individuals with weight, height, smoking status and genotype recorded included 66809 never-smokers, 43009 former smokers and 38913 current smokers. Waist circumference was available in 28 studies ( $\mathrm{n}=142381$ ), and hip circumference and waisthip ratio in 25 studies ( $n=139667$ ). Measures of fat mass and fat-free mass were provided by 10 studies $(\mathrm{n}=28231), \quad$ arm circumference by nine studies $(n=72536)$, and skinfolds by five studies $(n=7758)$. Finally, leptin and adiponectin were measured in nine studies (n=23630 and 19 191, respectively). Overall, $47 \%$ of the combined study population was male. The median age within the contributing studies ranged from 16-74 years. Descriptive statistics for each of the study populations are found in the supplementary material (see online supplementary table S1).

Minor allele frequency for $\mathrm{rs} 1051730 / \mathrm{rs} 16969968$ ranged between 0.31 and 0.36 . There was no strong evidence for deviation from the Hardy-Weinberg 
Equilibrium in any of the studies ( $p$ values all $\geq 0.09$, see online supplementary table S2).

\section{Mendelian randomisation analysis}

Table 1 shows the per-allele increases in each phenotype within each smoking status category. As previously shown, ${ }^{9}$ the increase in BMI was positive in neversmokers: $+0.35 \% \quad(95 \%$ CI $0.18 \%$ to $0.52 \%$; $\left.\mathrm{p}=6.38 \times 10^{-5}\right)$, non-significant in former smokers: $-0.14 \%$ (95\% CI $-0.34 \%$ to $+0.07 \%$; $p=0.19$ ) and significantly inverse in current smokers: $-0.74 \% \quad(95 \%$ CI $-0.96 \%$ to $-0.51 \% ; \mathrm{p}=2 \times 10^{-10}$ ). The full results for each contributing study are shown in online supplementary figure S1.

The waist circumference was higher per minor allele in never-smokers: $+0.23 \%$ (95\% CI $0.09 \%$ to $0.36 \%$; $\mathrm{p}=0.0012$ ), non-significantly related in former smokers $-0.07 \%$ (95\% CI $-0.24 \%$ to $0.09 \%$; $\mathrm{p}=0.37$ ) and lower in current smokers $-0.40 \%$ (95\% CI -0.57 to -0.22 $\left.\mathrm{p}=1.69 \times 10^{-5}\right)$ : differences among smoking groups were highly significant $\left(\mathrm{p}=3.85 \times 10^{-7}\right.$; see online supplementary figure S2. The per-allele effect on waist circumference in current smokers was about half the magnitude of that seen for BMI. After adjustment for $\log (\mathrm{BMI})$, the minor allele of rs1051730-rs16969968 was not associated with waist circumference in either never-smokers: $+0.01 \% \quad(95 \%$ CI -0.06 to $0.08 ; \mathrm{p}=0.72)$ or former smokers $+0.06 \%$ (95\% CI $-0.02 \%$ to $0.15 \%$; $\mathrm{p}=0.15)$. However, in current smokers, the minor allele was associated with a $0.14 \%$ (95\% CI $0.05 \%$ to $0.22 \% ; \mathrm{p}=0.003$ ) higher waist circumference after adjustment for $\log$ (BMI). Very similar results were seen in all three smoking status categories after waist was adjusted for $\log$ (weight) and height instead of for $\log (\mathrm{BMI})$. Effects of genotype on waist circumference were shown to differ between smoking status categories before adjustment $\left(\mathrm{p}=3.85 \times 10^{-7}\right)$ but only weakly after adjustment for $\log$ (BMI) $(p=0.102)$, and after adjustment for $\log$ (weight) and height $(p=0.018)$. Little heterogeneity of study results was evident $\left(\mathrm{I}^{2} \leq 25 \%\right.$ within all smoking groups). After restricting analysis to participants with BMI under $30 \mathrm{~kg} / \mathrm{m}^{2}$, we found that the percentage increases in waist circumference (after adjustment for $\log (\mathrm{BMI})$ ) were $0.04 \%(95 \%$ CI $-0.03 \%$ to $0.12 \%)$ for neversmokers, $0.03 \% \quad(95 \%$ CI $-0.06 \%$ to $0.13 \%)$ for ex-smokers and $0.12 \%$ (95\% CI $0.02 \%$ to $0.21 \%$ ) for current smokers: however, the test for difference in effects gave $\mathrm{p}=0.41$.

Unadjusted results for hip circumference were very similar to that seen for waist, both in direction and magnitude, in all smoking status groups (see online supplementary figure S3). However, after adjustment for $\log$ (BMI), effects were not apparent in any of the three groups, and nor was the interaction of gene and smoking status.

Results for the waist-hip ratio were similar to the BMI, waist and hip circumferences in direction but were smaller in magnitude: $+0.07 \%, 0.00 \%$ and $-0.08 \%$ increases in never-smokers, former smokers and current smokers, respectively $(\mathrm{p}=0.083$ for differences between smoking categories; see online supplementary figure S4). After adjustment for $\log (\mathrm{BMI})$, increases remained non-significant for never-smokers and former smokers $(-0.01 \%$ and $0.04 \%)$ but increased significantly among current smokers $(0.10 \%) \quad(\mathrm{p}=0.13$ for differences among smoking groups).

For several other phenotypes, per-allele decreases were observed in current smokers that exceeded those seen either in former or never-smokers (see online supplementary table S4). However, there was only statistical evidence for decreases among current smokers for arm circumference $\left(\mathrm{p}=8.4 \times 10^{-5}\right)$ and leptin $(\mathrm{p}=0.025)$, while the difference between smoking groups was only significant for arm circumference $\left(p=3.29 \times 10^{-4}\right)$. Both effects became non-significant after adjustment for $\log (\mathrm{BMI})$. Fat mass and fat-free mass, after adjustment by height, showed differences in effects by smoking group. These effects were more due to per-allele increases seen among never-smokers than decreases among current smokers.

Meta-regression analyses showed no clear evidence for associations between genotype and each adiposity phenotype being modified by sex: $p$ values exceeded 0.1 for all phenotypes, adjusted or unadjusted, apart from hip circumference. The per-allele decreases in hip circumference among current smokers appeared more marked among women $(\mathrm{p}=0.067)$, but this effect was no longer apparent after adjusting for BMI $(\mathrm{p}=0.51)$.

The mean difference in daily cigarette consumption was 0.77 among current smokers (95\% CI 0.67 to 0.88 , $\left.\mathrm{I}^{2}=17 \%\right)$.

\section{DISCUSSION}

This meta-analysis of 29 studies comprising almost 150000 participants with key adiposity phenotypes has demonstrated, first, that a variant associated with increased cigarette consumption was associated not only with lower BMI among current smokers, consistent with earlier findings, ${ }^{78}$ but also with lower waist and hip circumferences. Second, the inverse association of the variant with lower waist circumference among current smokers changed direction after adjusting for BMI. The variant was positively associated with waist circumference but associated neither with hip circumference after BMI adjustment nor waist-hip ratio. Our results suggest that for every copy of the minor allele associated with cigarette consumption (ie, increasing cigarette per day consumption by approximately one cigarette), waist circumference will be increased by $0.14 \%$ if BMI were to remain constant. This suggests a preferential redistribution towards central adiposity associated with higher cigarette consumption: this important finding is in keeping with our hypothesis and extends current observational data.

We also observed that none of the effects were modified by sex, contrary to our second hypothesis. Finally, 


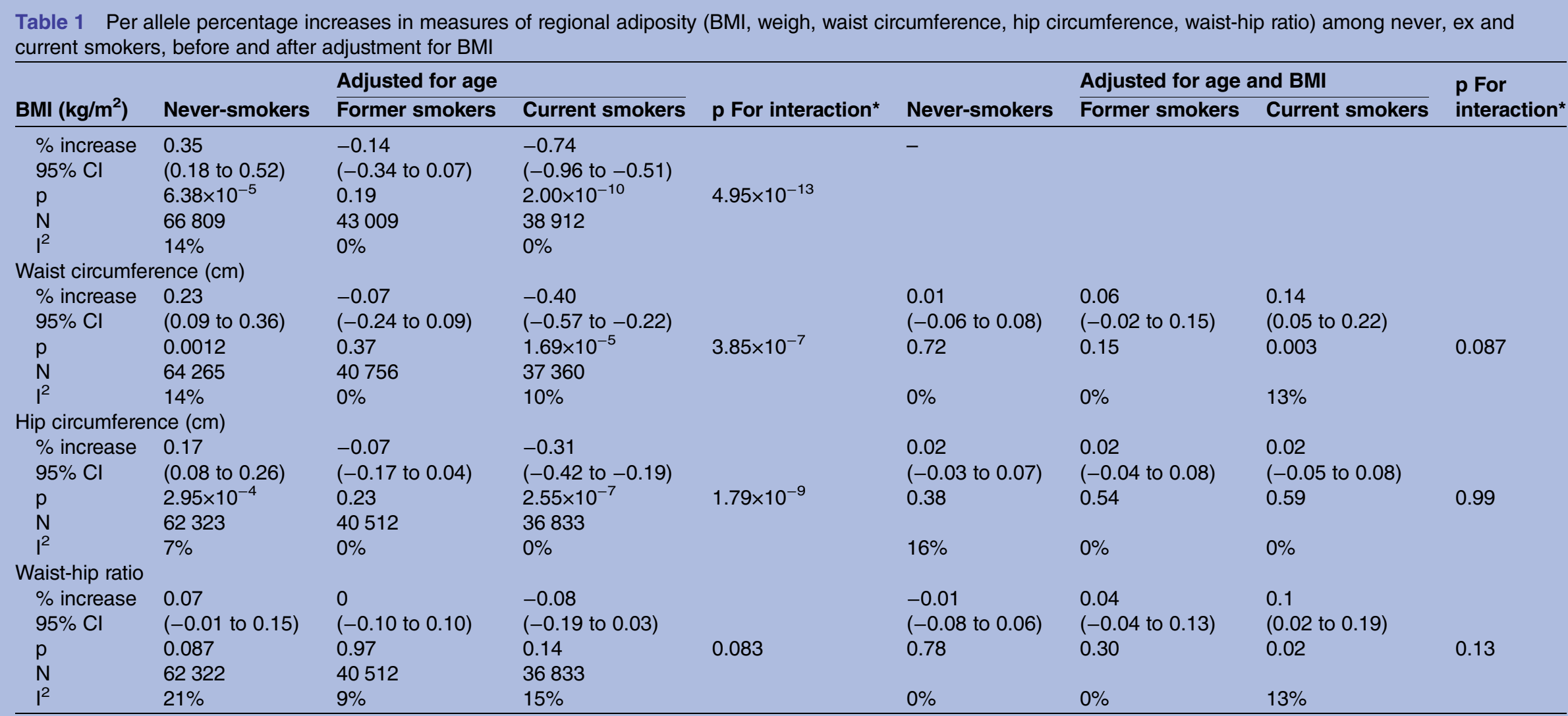

*Interaction assessed by assessing heterogeneity between effect estimates according to smoking status, with a fixed effects model.

BMI, body mass index. 
we have already noted among never-smokers an unexpected positive association of the gene variant with $\mathrm{BMI}^{9}$ : the current analysis demonstrates this same association with waist and hip circumferences. This occurred in the opposite direction to the inverse association of various adiposity measures with the gene variant seen in current smokers (before adjustment for BMI).

The analysis consisted of never, former and current smokers from a very wide spectrum of ages among the 29 studies. The sample size was very large for the primary phenotypes considered here. Participants were exclusively of self-reported European ancestry, and were mostly recruited in European countries. Data for direct measures of fat, such as fat mass, and the biomarker leptin were available for only about one-fifth of the participants whose weight, height, waist and hip were measured. Effects according to genotype for these phenotypes showed broadly similar results for the three smoking categories to those seen for BMI.

Mendelian randomisation has proved a powerful tool for eliciting causal associations between phenotypic measures. ${ }^{20}$ In the present analysis, Mendel's laws concerning random assignment of genotype should produce an unconfounded comparison between the genotype influencing smoking consumption and the outcomes of interest, namely anthropometric phenotypes. Furthermore, since this random assignment occurs at the very outset of life, the associations between genotype and anthropometric measures cannot be due to reverse causality. If the genotype only influences smoking consumption, and not the initiation of smoking, then the relationship between genotype and anthropometric outcomes would only be expected among smokers.

In fact, while the variant was associated with lower waist and hip circumferences among current smokers, it was associated with greater waist and hip circumferences among never-smokers. This suggests that the true effect among current smokers may be even greater than estimated. When we adjusted waist circumference for BMI, there was no association with the gene variant among never-smokers. The relative proportions of ever-smokers and never-smokers were not clearly associated with genotype in the CARTA consortium, as reported elsewhere. ${ }^{9}$

The reversal of the association between waist circumference and allele count from negative to positive among current smokers after adjustment for BMI may be consistent with alternative explanations. First, heavy smokers may have less muscle mass; however, no association between allele count and fat-free mass could be detected in our analysis among smokers. Second, the test for interaction for smoking status and allele count on waist circumference after adjustment was of weak statistical significance. Third, the adjustment of one measure of adiposity with another with which it is highly correlated may have caused a spurious association. We repeated our analysis for participants with BMI under 30 only, where the correlation was more modest, and obtained similar results, albeit with reduced evidence for an effect.
Stratification of our analyses by smoking status could, in theory, introduce bias by conditioning on a collider (rs1051730/rs16969968). ${ }^{21}$ This variant shows some evidence for association with smoking cessation (current vs former smoking).$^{22}$ While this is a possibility, no effect modifications of this variant with potential confounders by smoking status were demonstrated among 56625 participants in the HUNT study. ${ }^{8}$

Cross-sectional observational data from Switzerland has demonstrated that waist and hip circumferences were more strongly related to the number of cigarettes smoked per day than was BMI ${ }^{13}$ while in Scotland being a smoker was associated with greater central adiposity among women. ${ }^{12}$ In a Finnish longitudinal twin cohort study, smoking in adolescence predicted abdominal obesity in adulthood. ${ }^{11}$ Observational data are, however, prone to confounding and reverse causality, and the present study adds some evidence that the associations reported are likely to be causal.

Some observational studies have noted that low fat-free mass ${ }^{23}$ and bone mineral density ${ }^{24}$ were more common among smokers. The present analysis has not substantiated the association with fat-free mass, although our sample size was much more limited for this phenotype.

Our findings resonate with observational studies which have shown associations between smoking and risk of diabetes, ${ }^{17}{ }^{18}$ especially as analysis of the British Women's Heart and Health Study showed that abdominal adiposity was a stronger predictor of diabetes than was BMI. ${ }^{16}$ Waist circumference and waist-to-hip ratio were strongly associated, independently of BMI, with the risk of death among 359387 participants from nine countries in the European Prospective Investigation into Cancer and Nutrition. ${ }^{15}$ Therefore, the health hazards of smoking could well be enhanced or partly mediated through increasing abdominal adiposity. In addition, the desire of many smokers to use smoking as a means of weight control ${ }^{2}$ might be counterproductive if a loss of weight is accompanied by a relative increase in waist circumference: this possibility could be used in counselling people seeking to quit smoking.

People who quit smoking appear to be at increased risk of acquiring diabetes in the short term but this was not explained by weight gain in a Japanese population. ${ }^{25}$ This study took place almost exclusively among white European participants, and replication of the findings among other ethnic populations would be of great value. This is especially urgent on a global scale since smoking levels are increasing among several non-white ethnic groups, and this is seen to be partly responsible for increases in coronary heart disease mortality in Beijing, China, ${ }^{26}$ in Syria $^{27}$ and in Tunisia among women. ${ }^{28}$ In addition, increases in average waist circumference have been observed even when average BMI levels have remained constant, ${ }^{29}$ and metabolic disorders, especially diabetes, have increased in prevalence. ${ }^{30}$ It is thus possible that increased CHD mortality will be partly fuelled by increasing smoking levels. 
Mendelian randomisation studies have more potential than traditional observational epidemiological studies to establish causality for specific exposures, ${ }^{20}$ and they should now be used to investigate other impacts of smoking, in particular on pathways leading to type 2 diabetes, as well as on type 2 diabetes itself. The findings of this study could now be further tested by assembling data from randomised trials of smoking cessation, where postintervention data on measures of central adiposity are available. If confirmed, a tendency for smokers to acquire an 'apple shape' due to increasing central adiposity might provide a novel health promotion message to encourage smoking cessation, and appropriate new interventions should then be designed and evaluated as part of overall tobacco control policies in society.

\section{Author affiliations}

${ }^{1}$ School of Social and Community Medicine, University of Bristol, Bristol, UK ${ }^{2}$ Department of Primary Care and Population Health, UCL, London, UK ${ }^{3} \mathrm{MRC}$ Integrative Epidemiology Unit (IEU) at the University of Bristol, University of Bristol, Bristol, UK

${ }^{4}$ UK Centre for Tobacco and Alcohol Studies and School of Experimental Psychology, University of Bristol, Bristol, UK

${ }^{5}$ Department of Public Health, Faculty of Medicine, Norwegian University of Science and Technology, Trondheim, Norway

${ }^{6}$ Forensic Department, Research Centre Bröset, St Olav's University Hospital Trondheim, Trondheim, Norway

${ }^{7}$ Department of Endocrinology, St. Olavs Hospital, Trondheim University Hospital, Trondheim, Norway

${ }^{8}$ Department of Laboratory Medicine, Children's and Women's Health, The Faculty of Medicine, Norwegian University of Science and Technology, Trondheim, Norway

${ }^{9}$ Medical Genetics Section, Centre for Genomic and Experimental Medicine, Institute of Genetics and Molecular Medicine, University of Edinburgh, Edinburgh, UK

${ }^{10}$ Centre for Cognitive Ageing and Cognitive Epidemiology, University of Edinburgh, Edinburgh, UK

${ }^{11}$ Queensland Brain Institute, University of Queensland, Brisbane, Queensland, Australia

${ }^{12}$ Institute for Social and Economic Research, University of Essex, Colchester, UK

${ }^{13}$ Department of Public Health, Hjelt Institute, University of Helsinki, Helsinki, Finland

${ }^{14}$ Institute of Public Health and Clinical Nutrition, University of Eastern

Finland, Kuopio, Finland

${ }^{15}$ National Institute for Health and Welfare, Helsinki, Finland

${ }^{16}$ Department of Internal Medicine, Lausanne University Hospital, Lausanne, Switzerland

${ }^{17}$ Institute of Health Sciences, University of Oulu, Oulu, Finland

${ }^{18}$ Biocenter Oulu, University of Oulu, Oulu, Finland

${ }^{19}$ Centre for Environmental and Preventive Medicine, Wolfson Institute of

Preventive Medicine, Barts and the London School of Medicine and Dentistry, Queen Mary University of London, London, UK

${ }^{20}$ Population, Policy and Practice, UCL Institute of Child Health, University

College London, UK

${ }^{21}$ Department of Gerontology and Geriatrics, Leiden University Medical Center, Leiden, The Netherlands

${ }^{22}$ Netherlands Consortium of Healthy Ageing, Leiden, The Netherlands

${ }^{23}$ Research Centre for Prevention and Health, the Capital Region of Denmark, Denmark

${ }^{24}$ Novo Nordisk Foundation Center for Basic Metabolic Research, Section of Metabolic Genetics, Faculty of Health and Medical Sciences, University of Copenhagen, Copenhagen, Denmark

${ }^{25}$ Steno Diabetes Center, Gentofte, Denmark

${ }^{26}$ COPSAC, Copenhagen Prospective Studies on Asthma in Childhood, Herlev and Gentofte Hospital, University of Copenhagen, Copenhagen, Denmark

${ }^{27}$ Department of Biological Psychology, Netherlands Twin Register, VU University, Amsterdam, The Netherlands

${ }^{28}$ Faculty of Epidemiology and Population Health, London School of Hygiene

\& Tropical Medicine, London, UK

${ }^{29}$ Institute of Behavioural Sciences, University of Helsinki, Helsinki, Finland

${ }^{30}$ Folkhälsan Research Centre, Helsinki, Finland

${ }^{31}$ Wellcome Trust Sanger Institute, Wellcome Trust Genome Campus,

Cambridge, UK

${ }^{32}$ Institute for Molecular Medicine Finland (FIMM), University of Helsinki,

Finland

${ }^{33}$ The Medical and Population Genomics Program, The Broad Institute of MIT and Harvard, Cambridge, Massachusetts, USA

${ }^{34}$ Robertson Centre for Biostatistics, University of Glasgow, Glasgow, UK

${ }^{35}$ Institute of Cardiovascular and Medical Sciences, University of Glasgow,

Glasgow, UK

${ }^{36}$ MRC Unit for Lifelong Health and Ageing at UCL, London, UK

${ }^{37}$ Department of Environmental Medicine, Institute of Public Health, University of Southern Denmark, Odense, Denmark

${ }^{38}$ European Centre for Environment and Human Health, University of Exeter Medical School, Truro, UK

${ }^{39}$ Genetics of Complex Traits, University of Exeter Medical School, Exeter, UK

${ }^{40}$ Department of Psychological Medicine, University of Otago, Christchurch,

New Zealand

${ }^{41}$ Department of Pathology, University of Otago, Christchurch, New Zealand

${ }^{42}$ Institute for Clinical Research, University of Southern Denmark, Odense,

Denmark

${ }^{43}$ Department of Epidemiology, Biostatistics and Biodemography, Institute of

Public Health, University of Southern Denmark, Denmark

${ }^{44}$ University of Glasgow, Glasgow, UK

${ }^{45}$ Department of General Practice and Primary Health Care, University of Helsinki, Helsinki, Finland

${ }^{46}$ Unit of General Practice, Helsinki University Central Hospital, Helsinki, Finland

${ }^{47}$ Vasa Central Hospital, Vasa, Finland

${ }^{48}$ Population Health Research Institute, St George's University of London, London, UK

${ }^{49}$ Department of Clinical Experimental Research, Glostrup University Hospital, Glostrup, Denmark.

${ }^{50}$ Department of Clinical Medicine, Faculty of Health and Medical Sciences,

University of Copenhagen, Copenhagen, Denmark

${ }^{51}$ Department of Cardiology, Leiden University Medical Center, Leiden,

The Netherlands

${ }^{52}$ Durrer Center for Cardiogenetic Research, Amsterdam, The Netherlands

${ }^{53}$ Interuniversity Cardiology Institute of the Netherlands, Utrecht,

The Netherlands

${ }^{54}$ Centre for Population Health Research, School of Health Sciences and Sansom Institute of Health Research, University of South Australia, Adelaide, Australia

${ }^{55}$ South Australian Health and Medical Research Institute, Adelaide, South Australia, Australia

${ }^{56}$ Unit of Primary Care, Oulu University Hospital, Oulu, Finland

${ }^{57}$ Department of Children and Young People and Families, National Institute for

Health and Welfare, Oulu, Finland

${ }^{58}$ Department of Epidemiology and Biostatistics, MRC Health Protection Agency (HPA) Centre for Environment and Health, School of Public Health, Imperial College London, London, UK

${ }^{59}$ Department of Psychiatry, Lausanne University Hospital, Lausanne, Switzerland

${ }^{60}$ Institute for Molecular Medicine Finland FIMM, University of Helsinki, Helsinki, Finland

${ }^{61}$ Department of Epidemiology and Public Health, University College London, London, UK

${ }^{62}$ Division of Population Health Sciences, University of Dundee, Ninewells

Hospital and Medical School, Dundee, UK

${ }^{63}$ Medical Research Council Human Genetics Unit, Institute of Genetics and Molecular Medicine, University of Edinburgh, Edinburgh, UK

${ }^{64}$ Institute of Preventive Medicine, Bispebjerg and Frederikberg Hospitals,

The Capital Region, Copenhagen, Denmark

${ }^{65}$ Faculty of Medicine, BHF Glasgow Cardiovascular Research Centre, Glasgow, UK 
Acknowledgements 1958BC: Statistical analyses were funded by the Academy of Finland (Project 24300796 and SALVE/PREVMEDSYN). DNA collection was funded by MRC grant G0000934 and cell-line creation by Wellcome Trust grant 068545/Z/02. This research used resources provided by the Type 1 Diabetes Genetics Consortium, a collaborative clinical study sponsored by the National Institute of Diabetes and Digestive and Kidney Diseases (NIDDK), National Institute of Allergy and Infectious Diseases, National Human Genome Research Institute, National Institute of Child Health and Human Development, and Juvenile Diabetes Research Foundation International (JDRF) and supported by U01 DK062418. This study makes use of data generated by the Wellcome Trust Case-Control Consortium. A full list of investigators who contributed to generation of the data is available from the Wellcome Trust Case-Control Consortium website. Funding for the project was provided by the Wellcome Trust under the award 076113 . The work was supported by the Department of Health Policy Research Programme through the Public Health Research Consortium (PHRC). The views expressed in the publication are those of the authors and not necessarily those of the Department of Health. Information about the wider programme of the PHRC is available from http://phrc.Ishtm.ac.uk. Great Ormond Street Hospital/University College London, Institute of Child Health receives a proportion of funding from the Department of Health's National Institute for Health Research (NIHR) ('Biomedical Research Centres' funding). ALSPAC: We are extremely grateful to all the families who took part in this study, the midwives for their help in recruiting them, and the whole ALSPAC team, which includes interviewers, computer and laboratory technicians, clerical workers, research scientists, volunteers, managers, receptionists and nurses. The UK Medical Research Council and the Wellcome Trust (Grant ref: 092731) and the University of Bristol provide core support for ALSPAC. Ethics approval for the study was obtained from the ALSPAC Ethics and Law Committee and the Local Research Ethics Committee. Please note that the study website contains details of all the data that are available through a fully searchable data dictionary (http:// www.bristol.ac.uk/alspac/researchers/data-access/data-dictionary/). AET, MRM and MEF are members of the UK Centre for Tobacco and Alcohol Studies, a UKCRC Public Health Research: Centre of Excellence. Funding from the British Heart Foundation, Cancer Research UK, Economic and Social Research Council, Medical Research Council, and the National Institute for Health Research, under the auspices of the UK Clinical Research Collaboration, is gratefully acknowledged. This work was supported by the Wellcome Trust (grant number 086684) and the Medical Research Council (grant numbers MR/J01351X/1, G0800612, G0802736, MC_UU_12013/1, MC_UU_12013/6). BRHS: The British Regional Heart Study is a British Heart Foundation (BHF) Research Group. The BRHS has local (from each of the districts in which the study was based) and multicentre ethical committee approvals. BWHHS: The British Women's Heart and Health Study has been supported by funding from the British Heart Foundation (BHF) (grant PG/09/022) and the UK Department of Health Policy Research Programme (England) (grant 0090049). The BWHHS HumanCVD data were funded by the BHF (PG/07/131/24254). We thank all BWHHS participants, the general practitioners and their staff who have supported data collection since the study inception. Ethics approval was granted for the BWHHS from the London Multi-Centre Research Ethics Committee and 23 Local Research Ethics Committees. CaPS: The Caerphilly Prospective Study was conducted by the former MRC Epidemiology Unit (South Wales). The Caerphilly archive is now maintained by the School of Social and Community Medicine in Bristol University. We thank the Health and Social Care Information Centre (HSCIC) for helping us maintain long-term follow-up with the cohort. We thank all the men who have given their time to be participants in CaPS. Ethics approval was obtained from the South Glamorgan Area Health Authority, the Gwent REC and the South Wales Research Ethics Committee D. CHDS: The Christchurch Health and Development Study has been supported by funding from the Health Research Council of New Zealand, the National Child Health Research Foundation (Cure Kids), the Canterbury Medical Research Foundation, the New Zealand Lottery Grants Board, the University of Otago, the Carney Centre for Pharmacogenomics, the James Hume Bequest Fund, US NIH grant $\mathrm{MH} 077874$, and NIDA grant "A developmental model of gene-environment interplay in SUDs". (R01DA024413) 2007-2012. All phases of the study have received ethics approval from the regional Health and Disability Ethics Committee and all forms of data collection have been subject to the signed consent of research participants. Colaus: The CoLaus/PsyCoLaus study was supported by four grants of the Swiss National Science Foundation (\#105993, 118308, 139468 and 122661), two unrestricted grants from GlaxoSmithKline as well as by the Faculty of Biology and Medicine of the University of Lausanne. Colaus and PsyCoLaus were approved by the Institutional Ethics Committee of the University of Lausanne. Dan-MONICA: The Dan-MONICA10 was sponsored by The Danish Heart Foundation; the Danish Medical Research Council; The Danish Hospital Foundation of Medical Research, region of Copenhagen, the Faroe Islands and Greenland; The Danish Health Insurance Foundation; The Foundation of E. \& M. Wedel-Wedellsborg; Landsforeningen til Bekæmpelse af Kredsløbssygdomme; The Augustinus Foundation; The Becket Foundation; and The Foundation of senior registrar J. \& L. Boserup. All participants gave written consent and the study was conducted in accordance with the Second Helsinki Declaration and approved by the Ethics Committee for Copenhagen County. EFSOCH: The Exeter Family Study of Childhood Health (EFSOCH) was supported by the South West NHS Research and Development, Exeter NHS Research and Development, the Darlington Trust, and the Peninsula National Institute of Health Research (NIHR) Clinical Research Facility at the University of Exeter. The opinions given in this paper do not necessarily represent those of NIHR, the NHS or the Department of Health. Ethics approval was given by the North and East Devon Local Research Ethics Committee. ELSA: ELSA is funded by the National Institute on Aging in the US (R01 AG017644;R01AG1764406S1) and by a consortium of UK Government departments (including: Department for Communities and Local Government, Department for Transport, Department for Work and Pensions, Department of Health, HM Revenue and Customs and Office for National Statistics). ELSA has been approved by the National Research Ethics Service and all participants have given informed consent. FINRISK: This study was supported by the Academy of Finland Center of Excellence in Complex Disease Genetics (grant numbers 213506, 129680), the Academy of Finland (grant numbers 139635, 129494, 136895, 263836 and 141054), the Sigrid Juselius Foundation and ENGAGE-European Network for Genetic and Genomic Epidemiology, FP7-HEALTH-F4-2007, grant agreement number 201413. The 2002 and 2007 FINRISK surveys have been approved by the Coordinating Ethics Committee of the Helsinki University Hospital District. Each participant has given written informed consent. GEMINAKAR: The GEMINAKAR study was supported by grants from the Medical Research Fund, the Danish Diabetes Association, the NOVO Foundation and the Danish Heart Foundation. The study was approved by the relevant Danish Ethics Committee (baseline, S-VF-19970271) and Danish Data Protection Board (baseline, 1999-1200-441). All participants provided written informed consent.

Generation Scotland: Generation Scotland has received core funding from the Chief Scientist Office of the Scottish Government Health Directorates CZD/16/ 6 and the Scottish Funding Council HR03006. We are grateful to all the families who took part, the general practitioners and the Scottish School of Primary Care for their help in recruiting them, and the whole Generation Scotland team, which includes interviewers, computer and laboratory technicians, clerical workers, research scientists, volunteers, managers, receptionists, healthcare assistants and nurses. Genotyping of the GS:SFHS samples was carried out by the Genetics Core Laboratory at the Wellcome Trust Clinical Research Facility, Edinburgh, Scotland and was funded by the UK Medical Research Council (MRC). Ethics approval for the study was given by the NHS Tayside committee on research ethics (reference 05/s1401/89). GOYA: The GOYA study was conducted as part of the activities of the Danish Obesity Research Centre (DanORC, http://www.danorc.dk) and The MRC centre for Causal Analyses in Translational Epidemiology (MRC CAiTE). The genotyping for GOYA was funded by the Wellcome Trust (WT 084762). GOYA is a nested study within The Danish National Birth Cohort which was established with major funding from the Danish National Research Foundation. Additional support for this cohort has been obtained from the Pharmacy Foundation, the Egmont Foundation, The March of Dimes Birth Defects Foundation, the Augustinus Foundation and the Health Foundation. TSA was supported by the Gene Diet Interactions in Obesity (GENDINOB, http://www.gendinob.dk) postdoctoral fellowship grant. LP is funded by an MRC Population Health Scientist Fellowship (MR/J012165/1). The study was approved by the regional scientific ethics committee and by the Danish Data Protection Board. HBCS: The Helsinki Birth Cohort Study has been supported by grants from the Academy of Finland, the Finnish Diabetes Research Society, Samfundet Folkhälsan, Novo Nordisk Foundation, Finska Läkaresällskapet, Signe and Ane Gyllenberg Foundation, University of Helsinki, 
Ministry of Education, Ahokas Foundation and Emil Aaltonen Foundation. The research plan of the HBCS was approved by the Institutional Review Board of the National Public Health Institute and all participants have signed informed consent forms. Health2006/Health2008/Inter99: LLNH was supported by the Health Insurance Foundation (grant No. 2010 B 131). The studies have been approved by the Ethical Committee of Copenhagen. HUNT: Nord-Trøndelag Health Study (The HUNT Study) is a collaboration between the HUNT Research Centre (Faculty of Medicine, Norwegian University of Science and Technology NTNU), the Nord-Trøndelag County Council and the Norwegian Institute of Public Health. Use of data in this study was approved by the Regional Committee for Medical Research Ethics (Reference no. 2013/1127/ REK midt). Midspan: The Midspan Family Study was funded as part of the NHS Research and Development Cardiovascular Research Programme. Ethics approval was obtained from the Argyll and Clyde Health Board Local Research Ethics. NFBC: NFBC1966 and NFBC1986 received financial support from the Academy of Finland (project grants 104781, 120315, 129269, 1114194, 24300796, 141042 Center of Excellence in Complex Disease Genetics and SALVE), University Hospital Oulu, Biocenter, University of Oulu, Finland (75617), NHLBI grant 5R01HL087679-02 through the STAMPEED program (1RL1MH083268-01), NIH/NIMH (5R01MH63706:02), the European Commission (EUR0-BLCS, Framework 5 award QLG1-CT-2000-01643), ENGAGE project and grant agreement HEALTH-F4-2007-201413, EU FP7 EurHEALTHAgeing -277849, the Medical Research Council, UK (G0500539, G0600705, G1002319, PrevMetSyn/SALVE) and the MRC, Centenary Early Career Award. The DNA extractions, sample quality controls, biobank upkeeping and aliquoting was performed in the National Public Health Institute, Biomedicum Helsinki, Finland and supported financially by the Academy of Finland and Biocentrum Helsinki. We thank the late Professor Paula Rantakallio (launch of NFBCs) and Ms Outi Tornwall and Ms Minttu Jussila (DNA biobanking). The authors would like to acknowledge the contribution of the late Academian of Science Leena Peltonen. The University of Oulu Ethics Committee and the Ethical Committee of Northern Ostrobothnia Hospital District have approved the study. Participants provided written informed consent. NHANES: The National Health and Nutrition Examination Survey (NHANES) (http://www.cdc.gov/nchs/nhanes.htm) is a program of health surveys run by the National Center for Health Statistics, part of the Centers for Disease Control and Prevention in the USA. Data collection for NHANES was approved by the NCHS Research Ethics Review Board. Analysis of deidentified data from the survey is exempt from the federal regulations for the protection of human research participants. Analysis of restricted data through the NCHS Research Data Center is also approved by the NCHS ERB. The findings and conclusions in this paper are those of the author(s) and do not necessarily represent the views of the Research Data Center, the National Center for Health Statistics or the Centers for Disease Control and Prevention. NSHD: We are very grateful to the members of this birth cohort for their continuing interest and participation in the study. We would like to acknowledge the Swallow group at University College London, who performed the DNA extractions. This work was funded by the Medical Research Council [MC_UU_12019/1]. Ethics approval was given by the Central Manchester Research Ethics Committee. NTR: This study was supported by the European Research Council (ERC Starting Grant 284167 PI Vink), Netherlands Organization for Scientific Research (NWO: MagW/ZonMW grants 904-61-090, 985-10-002, 904-61-193, 480-04-004, 400-05-717, Addiction-31160008 Middelgroot-911-09-032, Spinozapremie 56-46414192), BBRMI-NL (Biobanking and Biomolecular Resources Research Infrastructure), VU University's Institutes for Health and Care Research and Neuroscience Campus Amsterdam. The NTR study was approved by the Central Ethics Committee on Research Involving Human Subjects of the VU University Medical Center, Amsterdam (IRB number IRB-2991 under Federalwide Assurance 3703; IRB/institute code 03-180), and all subjects provided written informed consent. PROSPER: The Prospective Study of Pravastatin in the Elderly at Risk (PROSPER) trial was supported by an investigator initiated grant from Bristol-Myers Squibb, USA. The study was conducted, analysed and reported independently of the company. The GWAS project PHASE has received funding from the European Union's Seventh Framework Programme (FP7/2007-2013) under grant agreement HEALTH-F22009-223004. A part of the genotyping was funded by The Netherlands Consortium for Healthy Ageing (NGI: 05060810). JWJ is an established clinical investigator of The Netherlands Heart Foundation (2001 D 032).
PROSPER was approved by the Argyll and Clyde Local Research Ethics Committee, the Glasgow Royal Infirmary Local Research Ethics Committee, Greater Glasgow Primary Care and Mental Health Research Ethics Committee, Lanarkshire Health Board Local Research Ethics Committee, Dumfries and Galloway Health Board Local Research Ethics Committee, Forth Valley Health Board Local Research Ethics Committee, METC board of Leiden University Medical Center and the Clinical Research Ethics Committee of The Cork Teaching Hospitals, and all participants gave written informed consent. Whitehall II: The Whitehall II study has been supported by grants from the Medical Research Council (K013351); British Heart Foundation; Health and Safety Executive; Department of Health; National Heart Lung and Blood Institute (NHLBI: HL36310) and National Institute on Aging (AG13196), US, $\mathrm{NIH}$; Agency for Health Care Policy Research (HSO6516); and the John D and Catherine T MacArthur Foundation Research Networks on Successful Midlife Development and Socio-economic Status and Health. MeKu is partially supported by the Economic and Social Research Council International Centre for Life Course Studies in Society and Health (RES-596-28-0001). MK is partially supported by the Medical Research Council and the Economic and Social Research Council. Ethics approval for the Whitehall II study was obtained from the University College London Medical School committee on the ethics of human research. Informed consent was gained from every participant.

\section{Collaborators Allan Linneberg.}

Contributors RWM, AET, TIAS, MRM and NS conceived the study and contributed to the writing of the manuscript. RWM conducted the final analyses. All other authors conducted individual study analyses and contributed to the writing of the manuscript.

Funding This research received no specific grant from any funding agency in the public, commercial or not-for-profit sectors.

Competing interests All authors have completed the Unified Competing Interest form at http://www.icmje.org/coi_disclosure.pdf (available on request from the corresponding author) and declare: support for the submitted work (LP, AW, DK: Medical Research Council, LJH, DMF: New Zealand Health Research Council, MAK: Jim and Mary Carney Charitable Trust, New Zealand Health Research Council, BHS: Scottish Government Chief Scientist Office) and financial relationships with any organisations that might have an interest in the submitted work in the previous three years (MP: grants from Swiss National Science Foundation and from GlaxoSmithKline, during the conduct of the study, TK: consulted for Pfizer in 2011-2015 on nicotine dependence, JK: grants from Academy of Finland, during the conduct of the study and personal fees from Pfizer, outside the submitted work). There are no other relationships or activities that could appear to have influenced the submitted work

Ethics approval The manuscript describes approval given for each of the 29 studies.

Provenance and peer review Not commissioned; externally peer reviewed.

Data sharing statement 1958BC: This study makes use of data generated by the Wellcome Trust Case-Control Consortium. A full list of investigators who contributed to generation of the data is available from the Wellcome Trust Case-Control Consortium website. The 1958 birth cohort data can be accessed via the UK Data Service (http://ukdataservice.ac.uk/). ALSPAC: Data used for this submission will be made available on request to the ALSPAC executive committee (alspac-exec@bristol.ac.uk). The ALSPAC data management plan (available here:http://www.bristol.ac.uk/alspac/researchers/ data-access/) describes in detail the policy regarding data sharing, which is through a system of managed open access. BRHS: We welcome proposals for collaborative projects and data sharing (http://www.ucl.ac.uk/pcph/ research-groups-themes/brhs-pub). For general data sharing enquiries, please contact Lucy Lennon (I.lennon@ucl.ac.uk). BWHHS: All BWHHS data collected are held by the research team based at the London School of Hygiene and Tropical Medicine for ongoing analysis. If you would like to collaborate with the BWHHS team, contact the study coordinator, AA (antoinette. amuzu@|shtm.ac.uk). Data and biological samples provided to the collaborators can only be used for the purposes originally stated and must not be used in any other way without re-application to the BWHHS team. 
No data should be passed on to any third party unless they were specified in the original application. CaPS: Data used for the Caerphilly Prospective study (CaPS) was made available by the CaPS access committee (Chair: Professor Kay Tee Khaw). More information about its managed access procedure is available on the study website (http://www.bris.ac.uk/

social-community-medicine/people/project/1392). CHDS: Data contributed for this submission are available on request from the CHDS (john.

horwood@otago.ac.nz).Colaus/PsyCoLaus: Data from the CoLaus/PsyCoLaus study can be requested according to the procedure described on the CoLaus website (http://www.colaus.ch/en/cls_home/cls_pro_home/cls-research-3. htm). ELSA: ELSA data are made available through the ESDS website (http:// www.elsa-project.ac.uk/availableData). FINRISK: Data used for this submission will be made available on request to the FINRISK Management Group, according to the given ethical guidelines and Finnish legislation. Generation Scotland: Data are available on request (access@generationscotland.org).GOYA females: An anonymised copy of the data used for this submission will be made available on request to the GOYA analysts after permission has been given by the DNBC executive committee (www.dnbc.dk). HBCS: Data used for this submission will be made available on request to the HBCS executive committee (johan.eriksson@helsinki.fi). Health2006/Health2008/Inter99: Data used for this submission can be made available on request to the Research Centre for Prevention and Health (http:// www.regionh.dk/fcfs/Menu/). Please contact LLNH (lise.lotte.nystrup. husemoen@regionh.dk) or AL (allan.linneberg@regionh.dk). HUNT: Data used from the HUNT Study for this submission will be made available on request to the HUNT Data Access Committee (hunt@medisin.ntnu.no). The HUNT data access information (http://www.ntnu.edu/hunt/data) describes in detail the policy regarding data availability. NFBC: Data used for this submission can be made available on request to Tuula Ylitalo (tuula.ylitalo@oulu.fi), Minna Mannikko (minna.annikko@oulu.fi) or M-RJ (m.jarvelin@imperial.ac.uk). NHANES: NHANES data can be accessed here: (http://www.cdc.gov/nchs/ nhanes.htm). The genotype used in this analysis is a restricted variable. Applications for access to these data must be made through the Research Data Center: (http://www.cdc.gov/rdc/). NSHD: The NSHD data are made available to researchers who submit data requests (tomrclha.swiftinfo@ucl.ac.uk). More information is available in the full policy documents (http://www.nshd.mrc.ac. uk/data.aspx). Managed access is in place for this study to ensure that use of the data is within the bounds of consent given previously by participants, and to safeguard any potential threat to anonymity since the participants are all born in the same week. NTR: Data used for this submission will be made available on request to the NTR committee (ntr@psy.vu.nl). Whitehall: Data from the Whitehall II study are made publicly available as described in the Whitehall II data sharing policy (http://www.ucl.ac.uk/whitehallll/datasharing).

TIAS, MRM and NS are joint senior authors.

Open Access This is an Open Access article distributed in accordance with the terms of the Creative Commons Attribution (CC BY 4.0) license, which permits others to distribute, remix, adapt and build upon this work, for commercial use, provided the original work is properly cited. See: http:// creativecommons.org/licenses/by/4.0/

\section{REFERENCES}

1. Doll R, Peto R, Boreham J, et al. Mortality in relation to smoking: 50 years' observations on male British doctors. BMJ 2004;328:1519.

2. Clark MM, Hurt RD, Croghan IT, et al. The prevalence of weight concerns in a smoking abstinence clinical trial. Addict Behav 2006;31:1144-52.

3. Luostarinen M, Tuovinen EL, Saarni SE, et al. Weight concerns among Finnish ever-smokers: a population-based study. Nicotine Tobacco Res 2013:15:1696-704.

4. Thorgeirsson TE, Geller F, Sulem P, et al. A variant associated with nicotine dependence, lung cancer and peripheral arterial disease. Nature 2008;452:638-42

5. Keskitalo K, Broms U, Heliovaara M, et al. Association of serum cotinine level with a cluster of three nicotinic acetylcholine receptor genes (CHRNA3/CHRNA5/CHRNB4) on chromosome 15. Hum Mol Genet 2009;18:4007-12.

6. Munafo MR, Timofeeva MN, Morris RW, et al. Association between genetic variants on chromosome $15 q 25$ locus and objective measures of tobacco exposure. J Natl Cancer Inst 2012;104:740-8.
7. Freathy RM, Kazeem GR, Morris RW, et al. Genetic variation at CHRNA5-CHRNA3-CHRNB4 interacts with smoking status to influence body mass index. Int J Epidemiol 2011;40:1617-28.

8. Asvold BO, Bjorngaard $\mathrm{JH}$, Carslake $\mathrm{D}$, et al. Causal associations of tobacco smoking with cardiovascular risk factors: a Mendelian randomization analysis of the HUNT Study in Norway. Int $J$ Epidemiol 2014;43:1458-70.

9. Taylor AE, Morris RW, Fluharty ME, et al. Stratification by smoking status reveals an association of CHRNA5-A3-B4 genotype with body mass index in never smokers. PLoS Genet 2014;10:e1004799.

10. Kim JH, Shim KW, Yoon YS, et al. Cigarette smoking increases abdominal and visceral obesity but not overall fatness: an observational study. PLOS ONE 2012;7:e45815.

11. Saarni SE, Pietilainen K, Kantonen S, et al. Association of smoking in adolescence with abdominal obesity in adulthood: a follow-up study of 5 birth cohorts of Finnish twins. Am J Public Health 2009;99:348-54.

12. Akbartabartoori M, Lean ME, Hankey CR. Relationships between cigarette smoking, body size and body shape. Int J Obes (Lond) 2005;29:236-43.

13. Clair C, Chiolero A, Faeh D, et al. Dose-dependent positive association between cigarette smoking, abdominal obesity and body fat: cross-sectional data from a population-based survey. BMC Public Health 2011;11:23.

14. Bigaard J, Tjonneland A, Thomsen BL, et al. Waist circumference, $\mathrm{BMI}$, smoking, and mortality in middle-aged men and women. Obes Res 2003;11:895-903.

15. Pischon T, Boeing H, Hoffmann K, et al. General and abdominal adiposity and risk of death in Europe. $N$ Engl J Med 2008;359:2105-20.

16. Taylor AE, Ebrahim S, Ben-Shlomo Y, et al. Comparison of the associations of body mass index and measures of central adiposity and fat mass with coronary heart disease, diabetes, and all-cause mortality: a study using data from 4 UK cohorts. Am J Clin Nutr 2010;91:547-56.

17. Wannamethee SG, Papacosta O, Whincup PH, et al. Assessing prediction of diabetes in older adults using different adiposity measures: a 7year prospective study in 6,923 older men and women. Diabetologia 2010;53:890-8.

18. Willi $\mathrm{C}$, Bodenmann $\mathrm{P}$, Ghali WA, et al. Active smoking and the risk of type 2 diabetes: a systematic review and meta-analysis. JAMA 2007;298:2654-64

19. Allison DB, Paultre F, Goran MI, et al. Statistical considerations regarding the use of ratios to adjust data. Int $J$ ObesRelat Metab Disord 1995;19:644-52.

20. Smith GD, Ebrahim S. 'Mendelian randomization': can genetic epidemiology contribute to understanding environmental determinants of disease? Int J Epidemiol 2003;32:1-22.

21. Cole SR, Platt RW, Schisterman EF, et al. Illustrating bias due to conditioning on a collider. Int J Epidemiol 2010; 39:417-20.

22. Taylor AE, Munafo MR, CARTA consortium. Commentary: does mortality from smoking have implications for future Mendelian randomization studies? Int J Epidemiol 2014;43:1483-6.

23. Castillo EM, Goodman-Gruen D, Kritz-Silverstein D, et al. Sarcopenia in elderly men and women: the Rancho Bernardo study. Am J Prev Med 2003;25:226-31.

24. Ward KD, Klesges RC. A meta-analysis of the effects of cigarette smoking on bone mineral density. Calcif Tissue Int 2001;68:259-70.

25. Oba S, Noda M, Waki K, et al. Smoking cessation increases short-term risk of type 2 diabetes irrespective of weight gain: the Japan Public Health Center-Based Prospective Study. PLoS One 2012;7:e17061.

26. Cheng J, Zhao D, Zeng Z, et al. The impact of demographic and risk factor changes on coronary heart disease deaths in Beijing, 19992010. BMC Public Health 2009;9:30.

27. Rastam S, Al AR, Maziak W, et al. Explaining the increase in coronary heart disease mortality in Syria between 1996 and 2006. BMC Public Health 2012;12:754.

28. Saidi O, Ben MN, O'Flaherty M, et al. Analyzing recent coronary heart disease mortality trends in Tunisia between 1997 and 2009. PLOS ONE 2013;8:e63202.

29. Chan JC, Malik V, Jia W, et al. Diabetes in Asia: epidemiology, risk factors, and pathophysiology. JAMA 2009;301:2129-40.

30. Danaei G, Finucane MM, Lu Y, et al. National, regional, and globa trends in fasting plasma glucose and diabetes prevalence since 1980: systematic analysis of health examination surveys and epidemiological studies with 370 country-years and 2.7 million participants. Lancet 2011;378:31-40. 


\section{Correction}

Morris RW, Taylor AE, Fluharty ME, et al. Heavier smoking may lead to a relative increase in waist circumference: evidence for a causal relationship from a Mendelian randomisation meta-analysis. The CARTA consortium. BMJ Open 2015;5:e008808. The author name Tarun Veer Singh Ahluwalia should be spelt Tarunveer Singh Ahluwalia, and the abbreviation is Ahluwalia TS. Also, the surname of Maiken Elvestad Gabrielsen is 'Gabrielsen' only so should be abbreviated to Gabrielsen ME as opposed to Elvestad Gabrielsen M.

BMJ Open 2015;5:e008808. doi:10.1136/bmjopen-2015-008808corr1

CrossMark 